\title{
El folclor de los pueblos y su incidencia en el currículo educativo
}

Folklore of the towns and its relation with the Educational Curriculum

Fortunato Diestra Salinas ${ }^{1}$, Virginia Asunción Cerafin Urbano ${ }^{1}$, Mara Sandra Rivera Galindo ${ }^{1}$, Nerio Janampa Acuña

\section{RESUMEN}

Objetivo: Conocer la relación del folclor de los pueblos y el currículo educativo de las instituciones educativas de la provincia de Huaura, Método: el método utilizado fue el nivel descriptivo correlacional, relacionando el folclor de los pueblos con el currículo educativo que se utiliza en las diferentes instituciones educativas. Resultado: se llegó a la conclusión que si existe relación entre el folclor de los pueblos y el currículo educativo, encontrándose una correlación moderada confiabilidad de 0,618.

Palabras clave: Folclor de los pueblos, currículo educativo.

\section{ABSTRACT}

Objective: To know the relationship of folklore of the peoples and the educational curriculum of the educational institutions of the province of Huaura, Method: the method used was the correlational descriptive level, relating the folklore of the people with the educational curriculum that is used in the different educational institutions. Result: it was concluded that if there is a relationship between the folklore of the people and the educational curriculum, a moderate reliability correlation of 0.618 is found.

Keywords: Peoples folklore, educational curriculum.

\section{INTRODUCCIÓN}

Una de las actividades de mayor importancia del docente es el dictado de clases, para ello utilizan todo las posibilidades que les puedan brindar las diferentes comunidades, tales como sus costumbres, los cuentos, los recursos naturales que pueden ser objeto de inspiración para la composición de una canción, poesía u otros, todos ellos naturalmente pertenecen al folclor de los pueblos. Por ello nace la inquietud de saber lo siguiente: ¿El docente hace uso del folclor de su comunidad como material didáctico? ¿Es provechoso hacer uso de estos recursos cuando se imparten conocimientos?, ¿Se relaciona en algo el folclor en la formación del estudiante?. Arévalo (2009) menciona que la educación debe partir del entorno y el contexto del alumno, siendo la música esencial para poder realizar los repertorios populares y folclóricas que cada día se pierden, los estudiantes serán los aliados estratégicos para seguir trasmitiendo esta manifestación cultural, de generación en generación. Gonzales (2011) manifiesta que Arguedas desarrolló un currículo contextualizado y pertinente que correspondía a la realidad para transformarla. En este tipo de currículo los estudiantes empiezan por describir o escribir sobre su pueblo: sus costumbres, su folklore, sus danzas, sus actividades agrícolas.

Ocaña (2015) manifiesta que cuando se habla de folclore se hace referencia al conjunto de elementos, ideas, tradiciones, formas de actuar y conocimiento popular que compone a la identidad de una comunidad determinada.

\footnotetext{
${ }^{1}$ Universidad César Vallejo. Lima Perú.
} 


\section{INTRODUCCIÓN}

Una de las actividades de mayor importancia del docente es el dictado de clases, para ello utilizan todo las posibilidades que les puedan brindar las diferentes comunidades, tales como sus costumbres, los cuentos, los recursos naturales que pueden ser objeto de inspiración para la composición de una canción, poesía u otros, todos ellos naturalmente pertenecen al folclor de los pueblos. Por ello nace la inquietud de saber lo siguiente: ¿El docente hace uso del folclor de su comunidad como material didáctico? ¿Es provechoso hacer uso de estos recursos cuando se imparten conocimientos?, ¿Se relaciona en algo el folclor en la formación del estudiante?. Arévalo (2009) menciona que la educación debe partir del entorno y el contexto del alumno, siendo la música esencial para poder realizar los repertorios populares y folclóricas que cada día se pierden, los estudiantes serán los aliados estratégicos para seguir trasmitiendo esta manifestación cultural, de generación en generación. Gonzales (2011) manifiesta que Arguedas desarrolló un currículo contextualizado y pertinente que correspondía a la realidad para transformarla. En este tipo de currículo los estudiantes empiezan por describir o escribir sobre su pueblo: sus costumbres, su folklore, sus danzas, sus actividades agrícolas.

Ocaña (2015) manifiesta que cuando se habla de folclore se hace referencia al conjunto de elementos, ideas, tradiciones, formas de actuar y conocimiento popular que compone a la identidad de una comunidad determinada. El folclore es justamente lo que construye la idiosincrasia de esa comunidad, haciendo que vistan, se alimenten, se diviertan, piensen y se muevan en su mundo de un modo particular y único. Por su parte, Tamayo (1997) manifiesta que el folclore es uno de los más vastos y perdurables nexos del pueblo peruano con su cultura ancestral: los mitos, tradiciones, cuentos, leyendas, danzas, poesías, canciones que tras un lento proceso de asimilación por el pueblo se enraízan y fructifican en todos los aspectos de la vida popular.
El currículo es la organización progresiva de los aprendizajes ordenados en grados, ciclo o niveles educativos y la disposición de las circunstancias que los hacen posibles por medio de las acciones de los sujetos que participan en su desarrollo que, posteriormente, presentan las habilidades, actitudes, conocimientos y comportamientos que constituyen los resultados del mismo (Galo, 2003). MINEDU (2016) consideró que el Currículo Nacional de la Educación Básica orienta los aprendizajes que se deben garantizar como Estado y sociedad. Debe ser usado como fundamento de la práctica pedagógica en las diversas instituciones y programas educativos, sean públicas 0 privadas; rurales o urbanas; multigrado, poli docente o unidamente; modelos y formas de servicios educativos. Asimismo, promueve la innovación y experimentación de nuevas metodologías y prácticas de enseñanza en las instituciones y programas educativos que garanticen la calidad en los resultados de aprendizaje.

Muchos autores consideran que el folclor de los pueblos, como ciencia tiene que responder a sus manifestaciones, todo lo que se dice, se hace es por iniciativa y costumbre de su poblador, en muchas comunidades las costumbres de convierten en leyes, y que son cumplidas de carácter obligatorio por todo los moradores. Por ello, el objetivo de la presente investigación fue conocer la relación del folclor de los pueblos y el currículo educativo de las instituciones educativas de la provincia de Huaura, Lima, Perú.

\section{MATERIAL Y METODOS}

La investigación se realizó en la ciudad de Huacho provincia de Huaura, en la sede de la universidad Nacional José Faustino Sánchez Carrión, en el periodo 2017, con estudiantes de maestría en pedagogía. La muestra fue de 60 estudiantes conformada por dos secciones, todos ellos trabajan en las distintas instituciones educativas de la provincia de Huaura ubicadas en provincias andinas y de la costa. La investigación fue de un nivel descriptivo correlacional, ubicado en el 
Tabla 1. Frecuencia y porcentaje de folclor de los pueblos y sus dimensiones

\begin{tabular}{|c|c|c|c|}
\hline Variable & Dimensiones & $\begin{array}{l}\text { Valor } \\
\text { IRango }\end{array}$ & $\begin{array}{l}\text { Porcentaje } \\
\%\end{array}$ \\
\hline \multirow{12}{*}{$\begin{array}{l}\text { Folclor de } \\
\text { los pueblos }\end{array}$} & \multirow{3}{*}{ Folclor de los pueblos } & Bajo & $33 \%$ \\
\hline & & Intermedio & $28 \%$ \\
\hline & & Alto & $38 \%$ \\
\hline & \multirow{3}{*}{ Cuentos y Leyendas } & Bajo & $30 \%$ \\
\hline & & Intermedio & $38 \%$ \\
\hline & & Alto & $32 \%$ \\
\hline & \multirow{3}{*}{ Costumbres } & Bajo & $45 \%$ \\
\hline & & Intermedio & $23 \%$ \\
\hline & & Alto & $32 \%$ \\
\hline & \multirow{3}{*}{ Alimentación } & Bajo & $23 \%$ \\
\hline & & Intermedio & $42 \%$ \\
\hline & & Alto & $35 \%$ \\
\hline \multirow{9}{*}{$\begin{array}{l}\text { Currículo } \\
\text { educativo }\end{array}$} & \multirow{3}{*}{ Currículo Educativo } & Bajo & $33 \%$ \\
\hline & & Intermedio & $53 \%$ \\
\hline & & Alto & $13 \%$ \\
\hline & \multirow{3}{*}{ Comunicación } & Bajo & $30 \%$ \\
\hline & & Intermedio & $63 \%$ \\
\hline & & Alto & $7 \%$ \\
\hline & \multirow{3}{*}{ Ciencias Sociales } & Bajo & $23 \%$ \\
\hline & & Intermedio & $60 \%$ \\
\hline & & Alto & $17 \%$ \\
\hline
\end{tabular}

En la Tabla 1, se tiene los resultados de cada uno de las variables y dimensiones evaluadas. La prueba de Kolmogorov - Smirnov indica que el resultado de ambas variables no sigue una distribución normal, en ambos casos siendo $(p<0,05)$, permitiendo de esta manera hacer uso de prueba no paramétrica, utilizando la correlación Spearman. El coeficiente de Spearman indica que existe relación entre el folclor de los pueblos y currículo educativo, hallándose una correlación moderada de 0,628 ( $p<0,000)$, confirmando que el folclor de los pueblos tiene relación significativa con el currículo educativo. El coeficiente de correlación de Spearman del folclor de los pueblos con la dimensión comunicación fue de 0.520 , lo que significa una correlación moderada $(p<0,000)$ afirmando que el folclor de los pueblos tiene relación significativa con la dimensión comunicación. De la misma manera, coeficiente de Spearman entre el folclor de los pueblos y la dimensión ciencias sociales fue moderada de 0,606 $(p<0,000)$ afirmando que el folclor de los pueblos tiene relación significativa con las ciencias sociales, lo que se muestra en la Tabla 2. 
Tabla 2. Coeficiente de correlación de Spearman entre el folclor de los pueblos y el currículo educativo, dimensión comunicación y Ciencias sociales

\begin{tabular}{|c|c|c|c|}
\hline \multirow[b]{2}{*}{ Variables } & \multicolumn{3}{|c|}{ Folclor de los pueblos } \\
\hline & $\begin{array}{l}\text { Coeficiente de } \\
\text { correlación }\end{array}$ & $\begin{array}{l}\text { Sig. } \\
\text { (bilateral }\end{array}$ & Interpretación \\
\hline Currículo educativo & $0,628^{* *}$ & 0,000 & $\begin{array}{l}\text { Altamente } \\
\text { significativo }\end{array}$ \\
\hline Comunicación & $0,520^{* *}$ & 0,000 & $\begin{array}{l}\text { Altamente } \\
\text { significativo }\end{array}$ \\
\hline Ciencias sociales & $0,606^{* *}$ & 0,000 & $\begin{array}{l}\text { Altamente } \\
\text { significativo }\end{array}$ \\
\hline
\end{tabular}

\section{DISCUSION}

Hemos tenido en cuenta a las investigaciones que nos anteceden en temas tratados con el nuestro, dentro de ello hemos encontrado cierta similitud con el trabajo de Arévalo (2009) que al realizar la investigación "Importancia del folklore musical como práctica educativa" concluye que la educación debe partir del entorno y el contexto del alumno, considerando que la música es esencial para poder realizar los repertorios populares y folclóricas que cada día se pierden. Estos resultados nos llevan reflexionar que, la música como recurso didáctico es de suma importancia para poder hacer extensivo las manifestaciones, de trasmitir y practicar una poesía con su entonación, pudiendo ser netamente una manifestación del lugar que es parte de su folclor.

Nuestro estudio está enfocado a entender cómo se relacionan estas manifestaciones folclóricas que se manifiestan en los diferentes pueblos con su aprendizaje de los estudiantes, entender como el docente hace uso de estos recursos, que logros consigue con ellos, especialmente con el canto, las leyendas, las costumbres, su alimentación, que muy bien pueden utilizar en las asignaturas de comunicación, ciencias histórico sociales, como en educación de ciencia y ambiente. Las evidencias recogidas en nuestro estudio así lo demuestran al arrojar que al relacionar el folclor de los pueblos con el currículo educativo, el 33\% manifiesta que es bajo, $28 \%$ es intermedio y el $38 \%$ de los encuestados manifiestan que es alto. De la misma manera se observan que los cuentos leyendas, tiene mayor frecuencia el intermedio, con $38 \%$, lo que necesita motivar a los demás docentes para hacer uso y se llegue al nivel alto. En costumbres, se tiene con mayor frecuencia el nivel bajo con $45 \%$ seguido, seguido del nivel alto con $32 \%$, lo que significa motivar a los demás docentes de la importancia del uso de esta dimensión y llegar al nivel alto. En ese mismo sentido, en el currículo de estudió se manifiesta que 30\% considera que la relación es bajo, $63 \%$ de los encuestados manifiestan que es intermedio.

Folclor de los pueblos tiene relación con el currículo educativo, debido a que en las instituciones educativas se programan a realizar una feria gastronómica, donde se presentan danzas de las diferentes regiones, así como también platos gastronómicos con la participación de los estudiantes y padres de familia, cuyo objetivo es conocer las riquezas folclóricas existentes en el país, con ello formar la identidad del estudiante. Este estudio no demuestra una correlación de moderada confiabilidad de 0,618.

Los resultados de nuestro estudio nos permiten concluir que predomina el uso de los cuentos y leyendas de los pueblos, especialmente en los estudiantes del nivel de educación inicial y primaria. Asimismo, se observa que, al relacionar el folclor de los pueblos y el currículo educativo falta motivar su uso para llegar al nivel alto. Las evidencias encontradas, nos permiten considerar que se deben plantear los contenidos temáticos desde la realidad del estudiante, propiciando plantear de esa manera los currículos regionales, basado netamente en la realidad de las comunidades que resultara ser, más efectivo el aprendizaje, y contribuyendo a su formación en su identidad del estudiante futuro poblador y ciudadano. 


\section{REFERENCIAS BIBLIOGRAFICAS}

Arévalo, A. (2009). Importancia del folklore musical como práctica educativa -Revista Electrónica LEEME. Recuperado el 9 de febrero de 2018 d e http://musica.rediris.es/leeme ISSN: 1575-9563.

Galo, C. (2003). El Currículo en el aula. Guatemala. Editorial Piedra Santa.

Gonzales, H (2011). "La experiencia pedagógica de José María Arguedas" Universidad Autónoma del Perú. Lima.

Mateo, L. (2003). Moderno Manual de Folklore y Danza del Perú, Editorial Ediciones "J.C", Perú: Lima.
Ocaña, E (2015). “El folclore social y su aporte al desarrollo del turismo cultural del cantón Ambato provincia de Tungurahua" tesis pregrado Universidad Técnica de AmbatoEcuador.

Sánchez, H y Reyes, C. (2005). Metodología y diseño en la investigación científica (4ta ed) Lima Perú: Editores Visión universitaria

Tamayo, W (1997). Folclore o cultura popular tradicional, Instituto Interamericano de derechos humanos.

Correo electrónico:

diestrasalinas@hotmail.com

Revisión de pares:

Recibido: 02-09-2019

Aceptado: 24-06-2019 